\title{
Arbor
}

\section{Significado presente del CSIC}

\author{
Miguel Ángel Garrido Gallardo
}

Arbor CLXVI, 653 (Mayo 2000), 1-15 pp.

Este artículo, elaborado en 1999, ofrece una panorámica de la realidad del Consejo Superior de Investigaciones Científicas en la actualidad. Repasa su organización, su volumen de publicaciones, sus indicadores de productividad, su capacidad de obtener recursos y, en suma, su lugar en el conjunto del sistema español de Ciencia y Tecnología, considerado en sí mismo y en relación con la Universidad. Como conclusión del repaso de datos, se afirma que, aunque la fórmula del CSIC, como organismo nacional e interdisciplinario, no es la única posible, la organización del Estado de las Autonomías, así como la evaluación de los datos con que se cuenta, la hacen vivamente recomendable. No hay, pues, sino que seguir con su desarrollo.

\section{Introducción}

El Consejo Superior de Investigaciones Científicas es el mayor organismo de que dispone el Estado español para realizar ciencia. Es multidisciplinario y sus institutos están diseminados por todo el territorio nacional, aunque todavía cerca de un $40 \%$ de ellos se encuentran en Madrid. Su situación al día de hoy es fruto de un ayer que, a medida que pasa el tiempo, se va historiando cada vez con mayor objetividad $^{1}$. Es también, sobre todo, una realidad volcada al futuro cuya virtualidad y límites son analizados en estas páginas.

Ciertamente, en el panorama mundial de los sistemas de Investigación y Desarrollo existen distintos modelos que entrañan cada uno sus correspondientes ventajas e inconvenientes. Son distintos el volumen 
que corresponde a lo público y lo privado en la realización de investigación científica y, por lo que hace a lo más próximo al sector público, existen opciones que vinculan la investigación básica casi exclusivamente al tejido de las universidades y existen otras (como la nuestra), que poseen un organismo del tipo del CSIC, el cual, a estos efectos (sólo a estos efectos), se puede definir como una universidad pública más, que se diferencia de las otras en que en vez de dedicarse a la docencia y a la investigación, se dedica casi por entero a esta última.

Como se sabe, el CSIC es la institución que nace tras la guerra civil de 1936-1939 para continuar la obra de desarrollo científico y cultural que llevaba a cabo la Junta de Ampliación de Estudios, vinculada a nombres como Santiago Ramón y Cajal o Ramón Menéndez Pidal. El paso del factotum de la Junta, Castillejo ${ }^{2}$ al del CSIC, Albareda ${ }^{3}$ ha sido juzgado de dos maneras: el CSIC es la institución franquista que corta la marcha progresiva de la Junta ${ }^{4}$ o el CSIC es el instrumento que en una época de ardor guerrero evita que el trabajo científico se estanque. De todos modos, en mi opinión, apenas tiene ya interés abordar una crítica ideológica de la cuestión y sí tiene mucho evaluar sus resultados. Eso es lo que intentaremos.

\section{Organización científica}

El CSIC se estructura actualmente en 8 áreas científico-técnicas, que son las que sigue la exposición de este volumen y que responden a una situación contemporánea de la ciencia, marcada por la transversalidad de las investigaciones. Más que el objeto formal físico, químico, biológico, etc., será el objeto material (recursos naturales, agricultura, materiales) lo que constituya el punto de unión. Precisamente en las intersecciones de unas ciencias con otras (interfases) es donde se suelen encontrar actualmente los mayores avances.

Naturalmente, según lo dicho, la clasificación estará abierta a sucesivas reestructuraciones a tenor de lo que vaya pidiendo la evolución de la ciencia. Creo, no obstante, que el presente diseño es suficientemente amplio y flexible como para que, con pequeñas matizaciones, pueda continuar por ahora. Biología y Biomedicina, Recursos Naturales, Ciencias Agrarias, Ciencia y Tecnologías Físicas, Ciencia y Tecnología de Materiales, Ciencia y Tecnología de Alimentos, Ciencia y Tecnología Quimicas y Humanidades y Ciencias Sociales comprenden un panorama verdaderamente universal. 


\section{Significado presente del CSIC}

En el CSIC, la unidad básica de carácter científico-administrativo es el Instituto que, para entendernos, equivale a la unidad Facultad de la estructura universitaria. El total comprende una red de 114 institutos, de los cuales 120 son centros propios y 36 centros mixtos constituidos mediante convenios con Comunidades Autónomas, Universidades $u$ otros entes de diverso rango.

Hay que advertir enseguida de la muy distinta importancia y tamaño de cada una de estas unidades. Como se verá al adentrarnos en la lectura, las hay de volumen e importancia notables y existen pequeños centros en formación o que responden a una tarea más o menos específica o marginal. Por ejemplo, el Instituto Hoffmeyer de Jaraíz de la Vera es tan sólo un pequeño centro cuya actividad se reduce principalmente a conservar, con ayuda de la Junta de Extremadura y otras instituciones locales, el legado y la revista sobre Historia de las armas que dejaron al CSIC un matrimonio de bibliotecarios daneses que se instalaron al final de su vida en España. La Escuela Española de Historia y Arqueología de Roma se constituye fundamentalmente como la continuación de la idea que tuvo D. Ramón Menéndez Pidal de crear en la urbe una instancia académica donde complementaran su formación sucesivas tandas de jóvenes historiadores y arqueólogos. En nada se parecen estas instituciones al Instituto de Historia, al Centro de Biología Molecular de Madrid o al Instituto de Microelectrónica de Barcelona, pongo por caso.

La distribución territorial es la siguiente: 46 institutos están en Madrid, 20 en Andalucía, 17 en Cataluña, 10 en la Comunidad Valenciana, 6 en Aragón, 5 en Castilla-León, 4 en Galicia, 2 en Asturias, 2 en el País Vasco, 1 en Cantabria, 1 en Navarra 1 en Baleares, 1 en Murcia, 1 en Castilla-La Mancha, y 1 en Canarias, además del citado de Extremadura y la Escuela de Historia y Arqueología de Roma.

Este panorama nos asoma a un beneficio actual, aún mas patente que cuando el CSIC se fundó, cuando vivimos en la España de las Autonomías. Los centros de la JAE estaban en Madrid. Es un acierto de Albareda el propósito que tuvo desde el principio de extender el CSIC a todo el territorio nacional. No cabe duda de que la unidad del organismo puede contribuir a vertebrar la dispersión derivada de la multiplicidad de localizaciones. Unas costosas instalaciones, localizadas en una Comunidad Autónoma, pero que alcanzan para las necesidades de toda la nación no tienen por qué ser multiplicadas por 17. El CSIC puede ser decisivo para que los esfuerzos se sumen y no se resten, para propiciar masas críticas adecuadas, mirando el conjunto de la Unión Europea que es donde a España le toca competir. 


\section{Miguel Ángel Garrido Gallardo}

Las sinergias que puede concitar el CSIC, sumándose a grupos universitarios y de otros Organismos Públicos de Investigación son también dignas de ser tenidas en cuenta. Un grupo científico del organismo que está al margen del sistema universitario, tiene una singular capacidad para servir de punto de encuentro de grupos universitarios de diferentes lugares. Así se viene haciendo con los centros mixtos, las unidades asociadas y la participación de profesores universitarios en proyectos del CSIC y, a la inversa, de científicos del CSIC en proyectos universitarios.

Si prestamos atención al hecho de que, a causa de la crisis de la natalidad, dentro de una década sobrará aproximadamente un tercio de las horas que el profesorado universitario invierte en dar clases, caeremos en la cuenta de hasta qué punto el CSIC puede servir para aprovechar disponibilidades y lograr una multiplicación notable del esfuerzo investigador de España. Desde luego, todo esto se puede hacer (y se hace) sin que sea necesaria la contribución del CSIC, pero, muchas veces, por sus características, nuestro Organismo convierte estos objetivos en más fáciles y hacederos.

\section{Producción Científica}

La producción científica medida en publicaciones controladas por el Institute for Scientific Information (ISI) de Philadelplia (Pennsylvania, USA) ${ }^{5}$ atestigua que en el período 1993-1997, el total de trabajos publicados de todas las áreas entre cuyos autores al menos uno tenía su sede institucional en España fue de 79.047. En ese momento y según esa referencia, el peso mundial de las publicaciones científicas nacionales representaba el $2,37 \%$ de las publicaciones científicas del mundo y otorgaba a España el undécimo lugar en la correspondiente clasificación (Tabla 1).

Pues bien, el número de publicaciones del CSIC en este período fue de 11.918, lo que supone el $0,36 \%$ de la producción científica mundial y el $15 \%$ de la española. Según mis noticias, el porcentaje español sigue ganando terreno en el conjunto mundial y el del CSIC, que andará ya en el 2000 por el $20 \%$ nacional, en el conjunto español.

Sabemos que todo dato meramente estadístico ha de ser debidamente ponderado, pero es cierto también que, por lo que hace a los grandes números, las mediciones bibliométricas resultan enormemente significativas. 


\section{Significado presente del CSIC}

TABLA 1

\begin{tabular}{|l|r|r|}
\hline & Número de trabajos & \% del total mundial \\
\hline Estados Unidos & 1.249 .520 & 37,41 \\
Reino Unido & 309.725 & 9,27 \\
Japón & 290.582 & 8,70 \\
Alemania & 269.588 & 8,07 \\
Francia & 205.826 & 6,16 \\
Canadá & 168.069 & 5,03 \\
Rusia & 123.281 & 3,69 \\
Italia & 123.062 & 3,68 \\
Australia & 89.622 & 2,68 \\
Países Bajos & 83.514 & 2,50 \\
España & 79.047 & 2,37 \\
India & 73.267 & 2,19 \\
Suecia & 63.904 & 1,91 \\
Suiza & 57.822 & 1,73 \\
Rep. Popular China & 57.135 & 1,71 \\
\hline
\end{tabular}

Aunque habitualmente, el dato numérico de publicaciones científicas se matiza cualitativamente con el índice de impacto o número de citas que reciben los trabajos consignados en la misma lista de revistas científicas solventes, no nos detendremos en él, porque creo que tal matización sólo vale para aquellas áreas cuya producción traspase un cierto volumen. En otros casos, resulta muy engañosa: si tuviéramos un solo gran investigador muy citado en una materia (18 citas), frente a mil, de toda laya y condición, que en Estado Unidos cultivaran la misma materia y consiguieran de media la mitad de impacto que nuestro supuesto científico excepcional, nos encontraríamos con que el «índice de impacto» español (18 citas, según el ejemplo) sería el doble que el de los Estados Unidos (9.000 citas, siguiendo también el ejemplo en cuestión). Lo mismo, por supuesto, puede ocurrir al revés y se puede referir a España con relación al resto del mundo o al CSIC con relación al conjunto español. Digamos, si acaso, que todo apunta a que, por lo general, las medias del CSIC están entre las mejores de España.

Ofreceremos, en cambio, los porcentajes de la producción científica total por especialidades (Tabla 2):

En esta tabla prescindo de las correspondientes entradas de Ciencias Sociales (que sí existen en el informe del ISI). Es de advertir que las medidas que poseemos al "respecto (y no digamos nada del área de Humanidades) son sumamente engañosas. De una parte, no existe 


\section{Miguel Ángel Garrido Gallardo}

un listado cerrado de revistas en inglés, como son la mayoría del ISI, que pueda ser significativo (poniendo un ejemplo extremo, pensemos que las revistas mejores de Filología Hispánica o Filología Francesa están casi todas escritas respectivamente en español o francés, como es natural). De otra parte, en Ciencias Sociales y en Ciencias Humanas (sobre todo), el resultado final de la investigación se suele entregar en forma de libro, a diferencias de lo que sucede en las ciencias duras donde los libros suelen reservarse para la divulgación. Resulta, pues, imposible medir cantidades tan heterogéneas mientras no se adopten las debidas cautelas de adaptación a los respectivos campos.

TABLA 2

\begin{tabular}{|l|c|c|}
\hline & $\begin{array}{c}\text { \% España } \\
\text { vs mundo }\end{array}$ & $\begin{array}{c}\text { \% CSIC } \\
\text { vs mundo }\end{array}$ \\
\hline Astrofísica & 4,13 & 0,6 \\
Ciencias Agrarias & 3,72 & 1,28 \\
Química & 3,47 & 0,72 \\
Matemáticas & 3,46 & 0,04 \\
Microbiología & 3,41 & 0,61 \\
C. Vegetales y Animales & 3,11 & 0,78 \\
Farmacología & 2,84 & 0,21 \\
Ecología/Medio Ambiente & 2,76 & 0,81 \\
Biología y Bioquímica & 2,53 & 0,42 \\
Física & 2,46 & 0,49 \\
Inmunología & 2,26 & 0,16 \\
Biología Molecular y Genética & 2,24 & 0,37 \\
Ingeniería & 2,16 & 0,26 \\
Neurociencias & 2,16 & 0,31 \\
Medicina Clínica & 2,09 & 0,04 \\
C. de Materiales & 1,93 & 0,66 \\
C. de la Tierra & 1,74 & 0,46 \\
\hline
\end{tabular}

La Tabla 2 servirá, en todo caso, para medir el diverso tamaño relativo de cada una las especialidades mencionadas, a partir del cual se pueden disponer correcciones de rumbos, coordinaciones, etc. según veremos en los respectivos capítulos monográficos.

También resulta ilustrativo el cuadro del número de publicaciones científicas de España en general y el CSIC en particular en el período que venimos examinando (Tabla 3). Sigo aplicando, no obstante, las mismas omisiones de la Tabla 2 por las razones dichas. 
TABLA 3

\begin{tabular}{|l|r|r|}
\hline & CSIC & España \\
\hline Química & 2.784 & 16.108 \\
Física & 1.953 & 10.356 \\
C. Vegetales y Animales & 1.634 & 7.432 \\
Biología y Bioquímica & 1.108 & 5.853 \\
C. Agrarias & 990 & 2.888 \\
Ingeniería & 758 & 6.820 \\
C. de Materiales & 750 & 2.215 \\
Ecología/Medio Ambiente & 611 & 2.067 \\
Microbiología & 467 & 2.603 \\
Neurociencia & 394 & 2.738 \\
C. de la Tierra & 392 & 1.496 \\
Biología Molecular y Genética & 368 & 2.223 \\
Medicina Clínica & 286 & 20.284 \\
Astrofísica & 233 & 1.608 \\
Farmacología & 163 & 2.236 \\
Multidisciplinar & 113 & 520 \\
Inmunología & 89 & 1.272 \\
Economía y Empresas & 43 & 539 \\
Matemáticas & 21 & 1.833 \\
Informática & 15 & 441 \\
\hline
\end{tabular}

Los datos de producción científica ofrecen algunas conclusiones inequívocas por lo que hace a la significación del CSIC en el conjunto de la $\mathrm{I}+\mathrm{D}$ española:

1. Los investigadores del CSIC, que constituyen el $6 \%$ del conjunto de los científicos españoles producen cerca del $20 \%$ de la ciencia que se hace en nuestro país.

2. El volumen de las áreas científicas del CSIC y del conjunto nacional no guardan siempre una proporción simétrica.

3. Aunque aquí no lo hayamos visto, en aquellas áreas cuyo volumen permite una adecuada medición de impacto, casi siempre la media del CSIC es superior a la media española y está entre las de los grupos más valorados ${ }^{6}$.

\section{Otros datos de productividad}

Otros indicadores referidos a 1998 también muestran resultados halagüeños para el CSIC: así, en la Memoria del año de referencia encontramos:

- Patentes en España: 386 (26\% de las del sector público)

- Patentes en países extranjeros: 224 (58\% de las 386) 
- Patentes licenciadas: 57

- Contratos/convenios: 505

- Cursos de especialización: 68

- Cursos de doctorado: 148

- Cursos del Fondo Social Europeo: 28

- Cursos de formación: 160

Añádase, en fin, la presencia del CSIC en certámenes y exposiciones (Tecnova, SIPAC, Expo 98,Biotechnica-Hannover) y completaremos un cuadro digno de consideración.

En cuanto a presencia internacional, el CSIC está presente en la European Heads of research Councils (Eurohorcs), en la European Science Foundation, en la Academia Europaea, en la Union Académique Internationale y en la mayoría de las uniones científicas federadas en el ICSU-CIUC (Consejo Internacional de Uniones Científicas). Existe una Delegación del CSIC en Bruselas (Oficina SOST) y numerosos científicos del Organismo participan en las más diversas comisiones internacionales.

En los años 1997/1998 se contabilizan en el CSIC 544 proyectos de I+D de la Unión Europea y 16 proyectos de la ESF más 204 proyectos de cooperación bilateral con 39 organismos de 22 países.

Completa este recuento la cooperación con 4 laboratorios franceses asociados (CSIC/CNRS), con el Centro Temático Europeo del Suelo (CSIC/EEA) y con la Agencia Española de Cooperación Internacional, sin contar las acciones más esporádicas en el marco de los Convenios Intergubernamentales a través del Ministerio de Asuntos Exteriores y la participación en las delegaciones españolas de conferencias de la ONU y la UNESCO.

El carácter nacional del CSIC facilita sin duda su carácter representativo en el orden internacional y, muy especialmente, en el ámbito de la Unión Europea de la que formamos parte. No cabe duda de que tal representatividad no debe ser la única de España ni excluyente, pero tampoco es dudoso que la extensión y diversidad coordinada de la actuación científica del CSIC ofrece óptimas posibilidades al respecto.

Hay que decir, con todo, que de los datos expuestos ahora, el de patentes no resulta satisfactorio, aunque el CSIC no sólo guarda la proporción que mantiene en publicaciones con el resto del sistema científico español, sino que la multiplica. No obstante, el CSIC, como uno de los motores que es de la I+D en España, tendrá que estar muy atento a superar esta debilidad. 


\section{Significado presente del CSIC}

\section{Obtención de recursos}

El CSIC viene participando de forma competitiva en las convocatorias, públicas y privadas, de proyectos de investigación: Plan Nacional (Programas Nacionales, PGC, FIS, Programa del INIA V, Planes de Investigación de las Comunidades Autónomas) y Programas Europeos. En este sentido, se constituye en una importante fuente de recursos para la investigación. La financiación está en armonía con las otras variables y, así, la obtención media de recursos del investigador del CSIC es tres veces superior a la media española como tres veces superiores son los resultados que de ellos se derivan. He aquí los números de 1997 y 1998:

\begin{tabular}{||l|c|c|c|c|}
\hline \multirow{2}{*}{} & \multicolumn{2}{|c|}{1997} & \multicolumn{2}{c||}{1998} \\
\cline { 2 - 5 } & NUMERO & MPTA & NUMERO & MPTA \\
\hline Plan Nacional & 1.074 & 5.028 & 1.145 & 6.134 \\
\hline Programas CCAA & 273 & 833 & 490 & 1.144 \\
\hline Programas Europeos & 470 & 4.990 & 496 & 7.155 \\
\hline
\end{tabular}

En el IV Programa Marco de la UE, el CSIC ha sido uno de las tres instituciones europeas que más recursos ha conseguido. En el año que llevamos del V, el CSIC ha obtenido más de 100 nuevos proyectos, lo que hace esperar un buen resultado final, a pesar de que la competencia ( $\mathrm{y}$, por tanto, las dificultades) aumentan de programa en programa.

También estos datos requieren su correspondiente matización. El volumen de recursos conseguidos están en relación con el tamaño del organismo unitario que es el CSIC. Como he dicho, en el seno de nuestro país, los resultados porcentuales resultan muy favorables. Habría que ponderarlos en el conjunto de la UE para poder obtener conclusiones válidas, puesto que tan alta clasificación quiere decir también obviamente que otros países concurren con agentes de menor tamaño y será necesario estudiar la incidencia que esa multiplicidad de instancias de tamaño medio tiene en los resultados. Un organismo que alcanza, por ejemplo, en conjunto el tamaño de otros seis, deberá ser comparado no con cada uno de los otros, sino con la suma de volumen similar.

Igualmente sale muy bien parado el CSIC con la comparación en cuanto a contratos o convenios con empresas y entidades públicas y 
privadas, aunque el crecimiento de la inversión del tejido industrial en $\mathrm{I}+\mathrm{D}$ ( $\mathrm{y}$, por consiguiente, también su repercusión en el CSIC) es, según común acuerdo, una de las asignaturas pendientes del desarrollo científico español.

El CSIC ha dispuesto diversas estrategias para su remedio: creación de plataformas industriales, apoyo al aprovechamiento industrial de la investigación, formación de alto nivel de personal para la industria, formación en innovación y tecnología. He ahí, sin duda, un amplio abanico de posibilidades que conectarán al CSIC con el tejido productivo e industrial. Nadie debe olvidar que el artificial enfrentamiento entre ciencia básica y ciencia aplicada está llamado a convertirse en recuerdo de otros tiempos. Nadie entienda, empero, que esto quiere decir que se opta por una de los dos extremos de un continuum que no debe tener (que no tiene) solución de continuidad.

\section{CSIC/Universisad}

Sin contar con los otros Organismos Públicos de Investigación, ni las demás instancias, sólo el conjunto de las universidades del Estado y el Consejo Superior de Investigaciones Científicas aportaron en 1998 más del $80 \%$ de la producción científica española y el $100 \%$ de las patentes surgidas en el sector público.

He dicho más arriba que a algunos efectos el CSIC puede ser considerado como una universidad pública más, casi la única estatal, por cierto, junto con la UNED y la «Menéndez Pelayo», que, como sabemos, tiene un carácter muy distinto. Sin embargo, hay diferencias ostensibles.

Una de estas diferencias es el sistema de selección de su personal científico investigador ${ }^{7}$. Al contrario que en la Universidad, los tribunales de concursos no se forman mediante la fórmula de dos profesores designados por la propia Facultad y tres elegidos por sorteo. En el CSIC, tras un proceso en el que intervienen diversos órganos colegiados, forman parte del tribunal profesores universitarios y científicos de otras instituciones junto con miembros del Instituto de que se trate. Según parece, la endogamia académica que asola la ciencia española desde la Ley de Reforma Universitaria de 1984, es mucho menos acusada en nuestro Organismo. Alrededor del $30 \%$ de los candidatos que han obtenido plaza de Científico Titular en los años 97 y 98 procedían de grupos (nacionales o no) ajenos al CSIC. Pienso, no obstante, que nada se salva totalmente, si se está inmerso en una corruptela general: 
sólo un sistema que permitiera a los aspirantes formados en el CSIC competir libremente en las universidades aseguraría una total apertura del CSIC a los candidatos formados en cualquier universidad.

El perfil medio del investigador que ha accedido a científico titular estos dos últimos años es ciertamente alto:

- 36 años de edad, habiendo transcurrido 12 desde que hizo la licenciatura y 6 ó 7 desde que obtuvo el grado de doctor.

- Con 33 meses de estudios postdoctorales en el extranjero.

- Con 19 publicaciones de alta calidad y notable impacto.

Fuera de comparaciones, el caso es que, en muchas ocasiones (que podrían ser muchas más), las universidades encuentran en el CSIC un adecuado complemento para llevar a cabo investigación de calidad. Naturalmente hay muchísimos proyectos científicos emprendidos por grupos de una universidad o de varias sin participación del CSIC, pero es verdad lo que hemos dicho más arriba de que la condición de la institución, volcada con exclusividad hacia la investigación, y su situación fuera de posibles rivalidades del circuito universitario, la tornan especialmente apta para convertirse en punto de encuentro de importantes iniciativas.

Volvamos a recordar las modalidades de cooperación existentes:

- Realización de proyectos conjuntos

- Cesión temporal de científicos del CSIC para trajar en universidades.

- Trabajo de grupos universitarios en Institutos del CSIC.

- Radicación de centros del CSIC en campus universitarios.

- Centros Mixtos.

- Unidades Asociadas.

Habría que detenerse en exponer el funcionamiento de los centros mixtos y las unidades asociadas, pero la extensión de este escrito no lo permite. Baste con recordar que hay ya una buena experiencia: los 3 Centros Mixtos y 50 Unidades Asociadas avalan estas íntimas formas de cooperación.

Por lo demás, es bien conocida la colaboración del científico del CSIC en la docencia universitaria, incluso a través de la figura de profesor asociado, tomada en su genuina significación. También son muchos los cursos universitarios de especialización o de magister que se imparten desde el CSIC o con la colaboración de su personal. Igualmente son numerosas las tesis doctorales dirigidas por científicos del CSIC. No es descartable, en fin, que desde el CSIC se pueda apoyar 
el diseño de nuevas licenciaturas acordes con los avances científicos y las nuevas demandas de la sociedad.

En la colaboración entre CSIC y Universidades, que debe sustanciarse en régimen de igualdad y reciprocidad, todo son ventajas. El volumen de más de 2.000 científicos de plantilla del CSIC (sin contar los cerca de 2.000 becarios en formación) no es comparable al de las universidades más grandes, pero si atendemos a su productividad, a la adecuación de infraestructuras y a la mejor dotación de personal de apoyo, puede ser suficiente para generar un entramado que triplique o cuadriplique el número de científicos que participen en los proyectos que se abordan desde o con él.

\section{El Color del cristal}

En pocos puntos existirá un acuerdo social tan unánime como en la afirmación de que, en estos umbrales del siglo XXI, España debe dar un paso al frente en investigación científica y tecnológica. El nivel de desarrollo alcanzado hace plausible la aspiración de situarse entre las primeras naciones del mundo y eso sólo se consigue mediante el avance en Ciencia y Tecnología.

Tampoco caben muchas opiniones diversas sobre la fórmula básica de esta operación: hay que invertir más recursos, hay que invertirlos adecuadamente y hay que séleccionar bien los científicos que deben hacerlos rendir. El factor humano es decisivo. Por eso, un problema como el de la endogamia académica representa una amenaza mucho mayor que la que aparece a primera vista. Por eso también, prejuicios democráticos que combaten contra la excelencia deben contarse entre los escollos más fuertes que sortear: aunque a nadie se le ocurre enviar a un cojo para correr la competición de los mil metros lisos (por mucho que haya sido elegido por sus compañeros), la convicción de que hay esferas necesariamente jerárquicas, también y sobre todo en la sociedad democrática, parece no haber calado suficientemente en muchos sectores académicos.

En todo caso, como resultado de lo visto, me parece que el Consejo Superior de Investigaciones Científicas está llamado a seguir siendo un factor decisivo para ese avance que debemos procurar. No digo que sea la única fórmula posible, pero afirmo, en cambio, que está dando tan buenos resultados como la mejor, razón por la que replanteársela (lo que a nadie se le ocurre) sería un disparate. 


\section{Significado presente del CSIC}

Es cierto, sin embargo, que el esplendor de algunos de los números expuestos pierde intensidad cuando se los observa más atentamente.

La obtención de recursos y la producción científica situada porcentualmente para el científico del CSIC en el 3 por 1 de la media del investigador español no sólo corresponde a la realidad de que su dedicación exclusiva a la investigación le otorga un notorio y notable plus de tiempo frente al profesor que debe atender la docencia. Ocurre también que la definición que se hace de investigador como todo personal académico con grado de doctor (por lo demás, común a otras naciones) contradice la más inmediata evidencia. ¿Cuántos docentes, de entre éstos, no tienen apenas contacto con la investigación?

Pero bueno sería que muchos de estos investigadores sin ejercicio fueran recuperados para la investigación a partir de las posibilidades de cooperación que el CSIC facilita de la forma múltiple y flexible que hemos visto.

Hay que admitir que el tamaño del CSIC y su dispersión por todo el territorio nacional entraña un esfuerzo enorme para su adecuada coordinación y gobierno. Merece, sin embargo, la pena mantenerlo así, pues, como hemos insistido, no cabe duda de que la propia estructura unitaria facilita los contactos, las interesantes iniciativas interdisciplinarias y el adecuado aprovechamiento de recursos con evitación de duplicados inútiles. Como se verá, diversos colaboradores de este número de Arbor coinciden en esta apreciación, como coinciden en que todavía hay que sacar más partido de las posibilidades que se derivan de aquí y que, por lo que nos dicen, están escasamente aprovechadas.

A pesar de lo dicho, como es natural, los candidatos que obtienen plaza en el CSIC no tienen por qué ser siempre científicamente mejores que sus colegas. Son iguales, mejores o peores que los compañeros que la obtienen en la Universidad. De todo habrá. Aunque llama la atención, desde luego, el perfil medio curricular antes señalado, hay que advertir que responde a un tapón que se había generado en los últimos tiempos y que la dotación de plazas de estos tres últimos años ha disuelto considerablemente. Estos datos deberán cambiar.

En efecto, no es medio exigible para la selección de científicos la situación heroica o excepcional. Deben arbitrarse nuevas medidas entre las que se contará la de los contratos quinquenales con sucesivas evaluaciones, de manera que se pueda modernizar y ampliar el sistema sin recargarlo de excesivo peso funcionarial. El nuevo Estatuto del CSIC, pendiente de aprobación, prevé esa posibilidad.

El CSIC puede facilitar que el potencial humano que la Universidad española está generando sea debidamente aprovechado. Nunca ha ha- 


\section{Miguel Ángel Garrido Gallardo}

bido tantos científicos y tan bien preparados. Por eso, se requieren instancias que faciliten su adecuada inserción en el sistema por sí mismo o por medio de sus relaciones en el entramado social. En este punto, estamos hablando de productividad, pero también -me gustaría decir que sobre todo- de asuntos humanos de primera importancia.

Dos palabras finales sobre los necesarios recursos económicos. También es precisa la reflexión al respecto. $\mathrm{Si}$ de la noche a la mañana llegáramos a invertir en España en investigación el 2\% del PIB que se ha propuesto como horizonte deseable (la media de la UE), dilapidaríamos el dinero de manera escandalosa. Es preciso ir preparando paso a paso las infraestructuras adecuadas y el contingente de científicos aptos, capaces de absorber un volumen tal de inversión. La realidad presente del CSIC aparece como uno de los elementos consolidados con medios para encauzar ese futuro.

La situación habla, en efecto, de futuro. Las cifras que se exponen en este número de Arbor revelan sólidas realidades con las que deberá contar el próximo Plan Nacional de I+D+I: los datos del CSIC. A ellos se habrán de añadir los de las universidades, más los de otros organismos públicos de Investigación y los de las instancias privadas.

Con todo, recuerdo que no he dicho que la fórmula del CSIC como organismo nacional y multidisciplinario, heredada de una historia que comienza a principios del siglo XX, sea teóricamente la única posible. Todo es según el color del cristal con que se mire. Insisto una vez más, sin embargo, en que, desde la perspectiva autonómica de la actual Constitución española y con la cuenta de resultados que presenta, aparece hoy como vivamente recomendable. No hay, pues, sino que seguir con su adecuado desarrollo en el siglo XXI.

\section{Notas}

1 J. M. SÁnchez Ron, Cincel, Martillo y piedra. Historia de la ciencia en España (siglos XIX y XX), Madrid, Taurus, 1999.

2 D. CASTILLEJo (comp.), Epistolario de Castillejo. I: Un puente hacia Europa 1896-1990.- II El espíritu de una época, 1910-1912, Madrid, Castalia, 1997 y 1998.

3 Vid. E. GuTiérRez Ríos, José María Albareda, una época de cultura española, Madrid, CSIC, 1970 y A. CASTILlo Genzor y M. TOMEo LaCRUe, Albareda fue así, Madrid, CSIC, 1971.

4 Cfr., p.e., M.J. SANTESMASES, «El legado de Cajal frente a Albareda: las ciencias biológicas en los primeros años del CSIC y los orígenes del CIB", en En torno a la 


\section{Significado presente del CSIC}

historia del CSIC, J.M.SÁNCHEZ RON (comp.), Arbor CLX, Madrid , julio-agosto, 1998, 305-332.

5 Los datos me han sido facilitados por la Dra. Matilde Sánchez Ayuso, Sudirectora General de Relaciones Internacionales del CSIC cuya amable ayuda deseo agradecer públicamente.

6 Cfr. M. SÁNCHEZ AYUSO, Análisis comparativo de la producción científica del CSIC. Informe de la Subdirección General de Relaciones Internacionales sobre la producción científica del Consejo Superior de Investigaciones Científicas, basado en datos del "Institute for Scientific Information» (ISI) de Philadelphia (Pennsylvania.USA).

7 Las escalas del Personal Científico Investigador del CSIC son actualmente tres: Científico Titular (equivalente del cuerpo de Profesores Titulares de Universidad), Investigador Científico (equivalente del extinto cuerpo de Profesores Agregados de Universidad) y Profesor de Investigación (equivalente del cuerpo de Catedráticos de Universidad). 